\title{
3D Antenna Structures Using Uniform Triangular Arrays for Efficient Full-Directional Multiuser Transmission
}

\author{
Jiyeong Yang $\mathbb{D}^{1},{ }^{1}$ Wonjae Ryoo $\left(\mathbb{D},{ }^{1}\right.$ Wonjin Sung $\left(\mathbb{D},{ }^{1}\right.$ Jeong-Ho Kim $\left(\mathbb{D},{ }^{2}\right.$ \\ and Jonghyun Park $\mathbb{1}^{3}$ \\ ${ }^{1}$ Department of Electronic Engineering, Sogang University, Seoul 04107, Republic of Korea \\ ${ }^{2}$ Department of Electronic and Electrical Engineering, Ewha Womans University, Seoul 03760, Republic of Korea \\ ${ }^{3}$ Advanced Standard R\&D Lab, LG Electronics, Seoul 07336, Republic of Korea \\ Correspondence should be addressed to Wonjin Sung; wsung@sogang.ac.kr
}

Received 11 February 2019; Accepted 30 September 2019; Published 5 November 2019

Guest Editor: Abhinav Kumar

Copyright (C) 2019 Jiyeong Yang et al. This is an open access article distributed under the Creative Commons Attribution License, which permits unrestricted use, distribution, and reproduction in any medium, provided the original work is properly cited.

In order to increase the system capacity of the $5 \mathrm{G}$ mobile communication system, multiple-input multiple-output (MIMO) transmission techniques using a large-scale array over the millimeter-wave band have attracted a great amount of attention. To cope with various types of receivers expected in 5G communications such as user equipment (UE) in small cells, indoor Internet-of-Things (IoT) devices at diverse locations, and drones performing aerial navigation, newer types of antenna arrays require all-directional transmission capability. Existing antenna structures with typical panel arrays, however, have restrictions on their transmission angles in both horizontal and vertical directions. In this paper, we propose to employ three-dimensional (3D) array structures composed of multiple triangular panels for efficient massive MIMO transmission of the next-generation wireless systems. We analyze beamforming characteristics of a uniform triangular array (UTA) suitable for such 3D array configurations and present a basic codebook applicable to UTAs. Using antenna structures with multiple UTA panels, multiuser transmission performance is evaluated to demonstrate the effectiveness of the proposal.

\section{Introduction}

Massive multiple-input multiple-output (MIMO) transmission effectively improves the data rate and the system capacity by increasing the spectral efficiency of $5 \mathrm{G}$ mobile communication systems [1-3]. By using the millimeter-wave frequency band, spacing between antenna elements is reduced to make the overall array size smaller, while accurate beamforming can be performed using a large number of antenna elements [4-6]. Codebook-based beam training methods have been proposed for transmission over the millimeter-wave [7], and beam management strategies using such training have been adopted for $5 \mathrm{G}$ new radio (NR) [8]. Existing codebook designs in the 3GPP standard are based on uniform linear arrays (ULAs) and uniform rectangular arrays (URAs), which utilize beamforming vectors chosen from the discrete Fourier transform (DFT) matrix [9], including the case for the 16-Tx URA in Release 14 specification [10]. Modifications to the conventional DFT-based codevectors have also been proposed to apply the existing codebooks to uniform circular arrays (UCAs) $[11,12]$.

Many different forms of transceivers need to be wirelessly connected to support machine-type communications (MTC), Internet-of-Things (IoT) devices, and vehicular-toeverything (V2X) communication for 5G NR. As locations of wireless devices become diversified, antenna arrays capable of transmitting and receiving beams over a wider range of directions are desired. While "full-directional" beamforming techniques to generate and track beams in an isotropic fashion become increasingly important, current antenna arrays have definite limitations to perform such beamforming. Three-dimensional (3D) array structures in different shapes can be utilized to overcome the limitations, with a large number of antenna elements to perform accurate beamforming to target directions. Traditional 3sector models using ULAs or URAs provide limited beamforming angles in both azimuth and zenith directions. Employing UCAs can overcome the drawback of degraded 
performance near sector boundaries by uniformly transmitting signals to all azimuth angles from circularly located antenna elements [13-17]. By vertically stacking the UCAs, a cylinder-type array structure can be constructed to add beam-tilting controllability over a certain range of zenith angles [18]. To expand the range of signal transmission over all azimuth and zenith angles, an array in spherical shape can be used for uniform and stable beamforming to all directions [19]. In an effort to construct antenna arrays capable of transmitting signals over the 3D space, geodesic domes have been used for satellite communications by dividing regular polyhedrons [20-23].

Designing 3D antenna array structures requires practical considerations including beamforming methodology, channel feedback signaling strategy, and hardware implementation complexity. Although full digital beamforming enables accurate and flexible beamforming to target directions, it requires the connection of an RF chain to each antenna element, which is not only costly but also limited by the space constraint of the array. To solve this problem, an array composed of subarrays or panels of antenna elements is desired, for which a plurality of antennas are bundled as a panel to be connected to the same RF chain [24-26]. Therefore, it is necessary to determine the basic panel shape for efficient construction of 3D arrays. While the URA is the most widely used type of antenna panels, building 3D arrays composed of URAs only involves design challenges. An alternative way of $3 \mathrm{D}$ array construction is to use antenna subarrays in triangular shape with even spacing between adjacent antenna elements, referred to as the uniform triangular array (UTA). Despite some earlier analyses on the physical patch design for UTAs $[27,28]$, utilization of triangular arrays for $3 \mathrm{D}$ beamforming has not been extensively investigated.

In this paper, we propose to utilize UTAs to construct multipanel 3D arrays for efficient MIMO transmission and present design examples to be used as next-generation base station antenna arrays. A specific array structure with a different number of panels can be chosen to be used under the given conditions of the transceiver, such as its location, cell size, and the user distribution. We first analyze the beam pattern produced by a single UTA and present the basic beamforming vectors applicable to the UTA for beam-tilting purposes. These vectors can be combined for coherent transmission to the target user from multiple panels constituting the whole array. They can also be used for multiuser signal transmission by appropriately allocating a set of UTA panels to each user. The arrays and corresponding beamforming vectors are applied to different transmission environments to demonstrate the usefulness of the proposal. The paper is organized as follows. Section 2 explains signal and system models, followed by the measures used for performance evaluation. In Section 3, beam characteristics of the UTA are analyzed, and beamforming vectors for the single UTA as well as for 3D multipanel arrays are presented. Array structures consisting of multiple UTAs are given in Section 4, and performance evaluation results are shown in Section 5. Conclusions are given in Section 6.

\section{System Model}

The UTA in consideration has the form of the equilateral triangle with constant spacing between the adjacent antenna elements in it. A UTA with 15 antenna elements is shown in Figure 1(a), where the number of elements in 5 antenna rows is $1,2,3,4$, and 5, respectively. Generalising this type of antenna placement, the $l$-th antenna row of the panel includes $l$ antenna elements. For the triangular panel with $L$ antenna rows, the number of antennas in the UTA is $N=L(L+1) / 2$. If the entire antenna array structure is composed of $M$ UTA panels, the number of total antenna elements becomes $M_{T}=M N$. To compare the beam patterns, we consider the conventional rectangular array with a similar number of antennas, which is the URA with 16 antenna elements, as shown in Figure 1(b). Parameters $\alpha$ and $\beta$ shown in Figure 1 represent the horizontal and vertical angles used for visualisation of beam patterns.

An example of the 3D antenna structure and related coordinate parameters are shown in Figure 2, where 20 UTA panels constitute the icosahedron-based antenna array. The centre of the $3 \mathrm{D}$ array is called origin and denoted by $O$. The target user equipment (UE) is denoted by $U$. Symbol $A_{m, n}$ denotes the location of the $n$-th antenna element in the $m$-th UTA panel. The direction of the UE can be represented in polar coordinates using the azimuth angle $\Phi$ and zenith angle $\Theta$. Also, the direction of $A_{m, n}$ from the origin is represented by the azimuth angle $\phi_{m, n}$ and zenith angle $\theta_{m, n}$. Denoting the distance from the origin to $A_{m, n}$ by $r_{m, n}$, the location vector for the $n$-th antenna element in the $m$-th panel is expressed as

$$
A_{m, n}=\left(r_{m, n} \cos \phi_{m, n} \sin \theta_{m, n}, r_{m, n} \sin \phi_{m, n} \sin \theta_{m, n}, r_{m, n} \cos \theta_{m, n}\right) \text {, }
$$

similarly, the UE location vector can be written as $U=(\rho \cos \Phi \sin \Theta, \rho \sin \Phi \sin \Theta, \rho \cos \Theta)$, where $\rho$ is the distance from the origin to the UE.

The signal transmitted from the antenna array with $M$ panels and received by the $k$-th UE can be written as

$$
\mathbf{y}_{k}=\sum_{m=1}^{M} \mathbf{H}_{k, m} \mathbf{w}_{k, m} s_{k}+\mathbf{I}_{k}+\mathbf{n}_{k},
$$

where $\mathbf{y}_{k}$ is the received vector, $\mathbf{H}_{k, m}$ is the channel matrix for the $m$-th panel, $\mathbf{w}_{k, m}$ is the beamforming vector for the $m$-th panel, and $s_{k}$ is the data symbol. Intercell interference and complex Gaussian noise vectors are denoted by $\mathbf{I}_{k}$ and $\mathbf{n}_{k}$, respectively. The signal model can be extended to the case when beams are formed to multiple target UEs as

$$
\begin{aligned}
\mathbf{y}_{k} & =\sum_{m=1}^{M} \mathbf{H}_{k, m} \mathbf{W}_{m} \mathbf{s}+\mathbf{I}_{k}+\mathbf{n}_{k} \\
& =\sum_{m=1}^{M} \mathbf{H}_{k, m} \mathbf{w}_{k, m} \boldsymbol{s}_{k}+\sum_{u \neq k} \sum_{m=1}^{M} \mathbf{H}_{k, m} \mathbf{w}_{u, m} \mathbf{s}_{u}+\mathbf{I}_{k}+\mathbf{n}_{k},
\end{aligned}
$$

where $\mathbf{y}_{k}$ is again the received vector for the $k$-th UE and $\mathbf{s}=\left[s_{1}, s_{2}, \ldots, s_{K}\right]^{T}$ is the data vector for $K$ UEs serviced by 


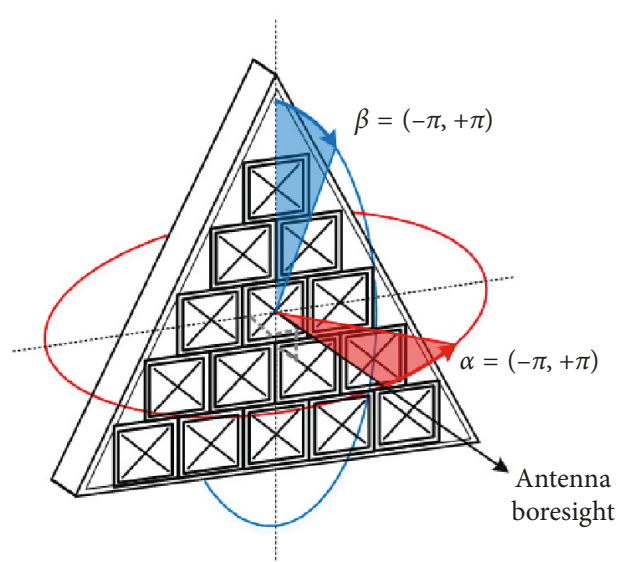

(a)

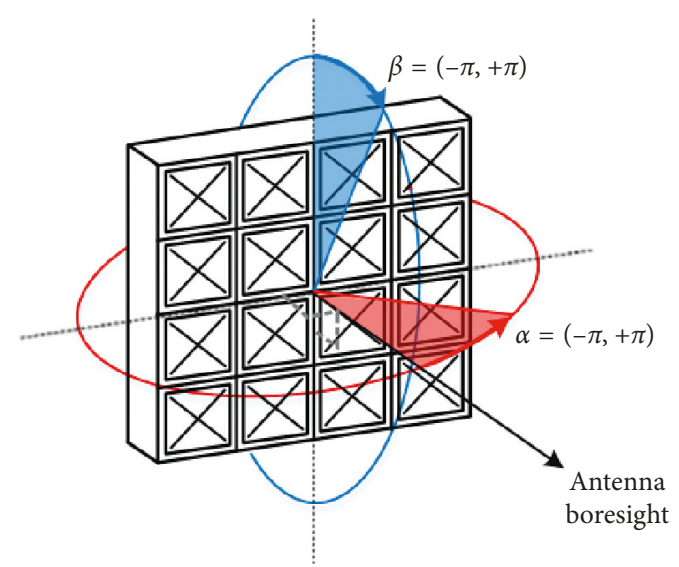

(b)

FIgURE 1: Comparison of antenna arrays. (a) UTA and (b) URA.

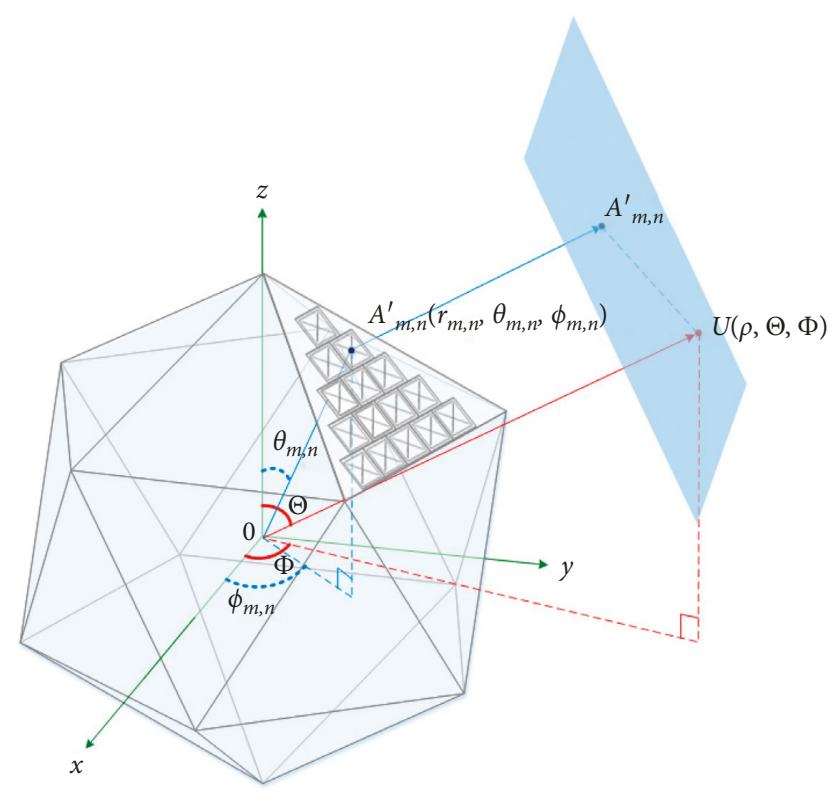

FIGURE 2: 3D antenna structure composed of UTA panels and coordinate information.

the transmit antenna array. Beamforming matrix $\mathbf{W}_{m}=$ $\left[\mathbf{w}_{1, m}, \mathbf{w}_{2, m}, \ldots, \mathbf{w}_{K, m}\right]$ includes the beamforming vectors for $K$ UEs.

When multiuser data are simultaneously transmitted, the beams intended for the other UEs act as the interference and the corresponding interference signal is represented by the second term in equation (3). Combining the effect of interuser interference, intercell interference, and the noise, the signal-to-interference-plus-noise-ratio (SINR) for the $k$-th UE becomes

$$
\Gamma_{k}=\frac{\left|\sum_{m=1}^{M} \mathbf{H}_{k, m} \mathbf{w}_{k, m}\right|^{2}}{\left|\sum_{u \neq k} \sum_{m=1}^{M} \mathbf{H}_{k, m} \mathbf{w}_{u, m}\right|^{2}+\left|\mathbf{I}_{k}\right|^{2}+N_{0}},
$$

where $N_{0}$ is the noise power. Summing up the individual data rate $R_{k}=\log _{2}\left(1+\Gamma_{k}\right)$ for $K$ UEs, the sum rate of the system is represented by

$$
R=\sum_{k=1}^{K} \log _{2}\left(1+\Gamma_{k}\right)
$$

The antenna gain pattern applied to each antenna element follows the 3GPP standard model in [29] given as

$$
G\left(\varphi_{v}, \varphi_{h}\right)=-\min \left\{-\left[G_{v}\left(\varphi_{v}\right)+G_{h}\left(\varphi_{h}\right)\right], 30\right\}[\mathrm{dB}],
$$

where $G_{v}\left(\varphi_{v}\right)=-\min \left\{\left(\varphi_{v} / 65^{\circ}\right)^{2}, 30\right\}$ is the vertical gain and $G_{h}\left(\varphi_{h}\right)=-\min \left\{\left(\varphi_{h} / 65^{\circ}\right)^{2}, 30\right\}$ is the horizontal gain. Arguments $\varphi_{v}$ and $\varphi_{h}$ represent the vertical and horizontal deviation angles from the antenna boresight, respectively.

\section{Uniform Triangular Array}

We generate the beam pattern of the UTA using the array shown in Figure 1(a). We assume no phase shifting is applied to the antenna elements and the beam direction is identical to the boresight of the array. The beam pattern is obtained by calculating the signal power at different values of the horizontal and vertical angles. Figure 3(a) indicates the horizontal beam pattern obtained by changing $\alpha$ for given values of $\beta$. Also, Figure 3(b) indicates the vertical beam pattern obtained by changing $\beta$ for given values of $\alpha$. For comparison, similar beam patterns are evaluated for the URA using the array shown in Figure 1(b), and the results are plotted in Figure 4. Note that the horizontal and vertical patterns for the URA are of the same shape due to the symmetry of the array. Although the patterns for the UTA and URA are not identical, general beam shapes including the $3 \mathrm{~dB}$ beamwidth and the magnitudes of sidelobes are similar.

The beam patterns when the target beam direction deviates from the array boresight are also compared for the two arrays. Figure 5(a) shows the peak power observed at the target direction when the beam direction deviation angle $\theta$ increases from 0 , assuming the unit power is transmitted from each antenna element. The peak power tends to decrease as $\theta$ becomes large due to the decreasing gain of unit antennas described in equation (6). However, very similar peak power values are observed for the two arrays for all 


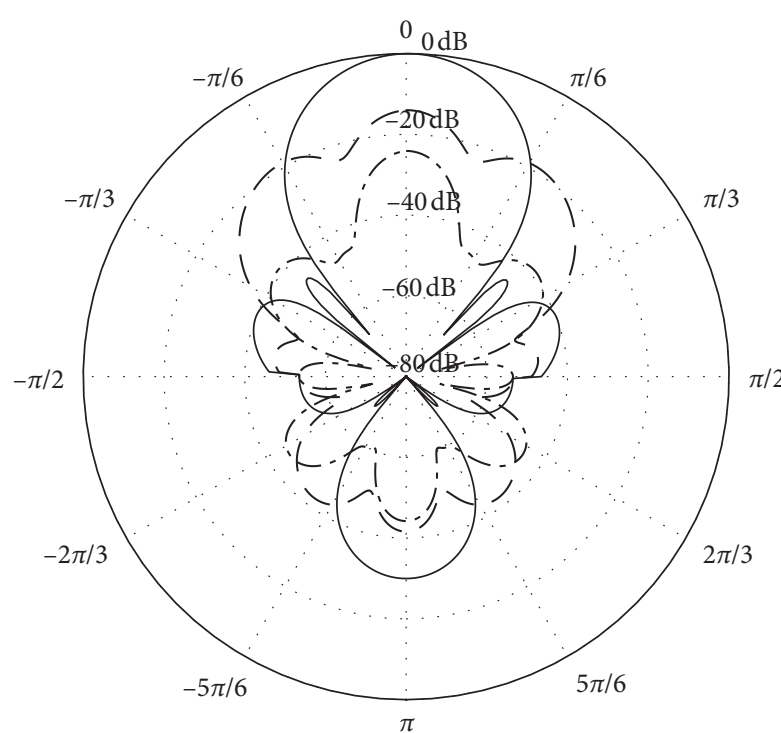

$$
\begin{aligned}
-\beta & =90^{\circ} \\
--\beta & =60^{\circ} \\
--\beta & =30^{\circ}
\end{aligned}
$$

(a)

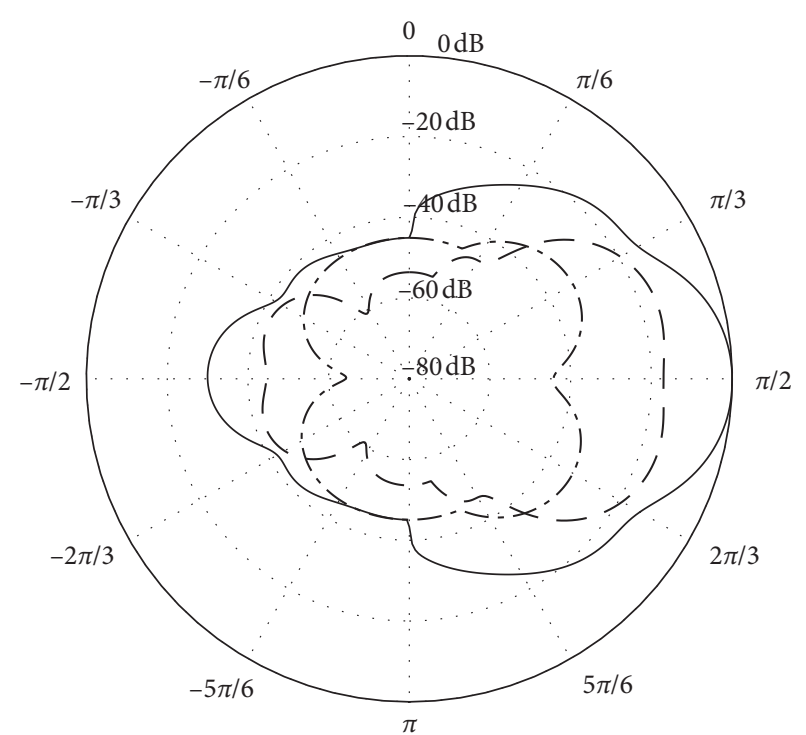

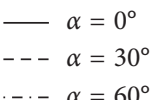

FIgURE 3: UTA beam pattern. (a) Horizontal pattern: $-\pi \leq \alpha \leq \pi$; (b) vertical pattern: $-\pi \leq \beta \leq \pi$.

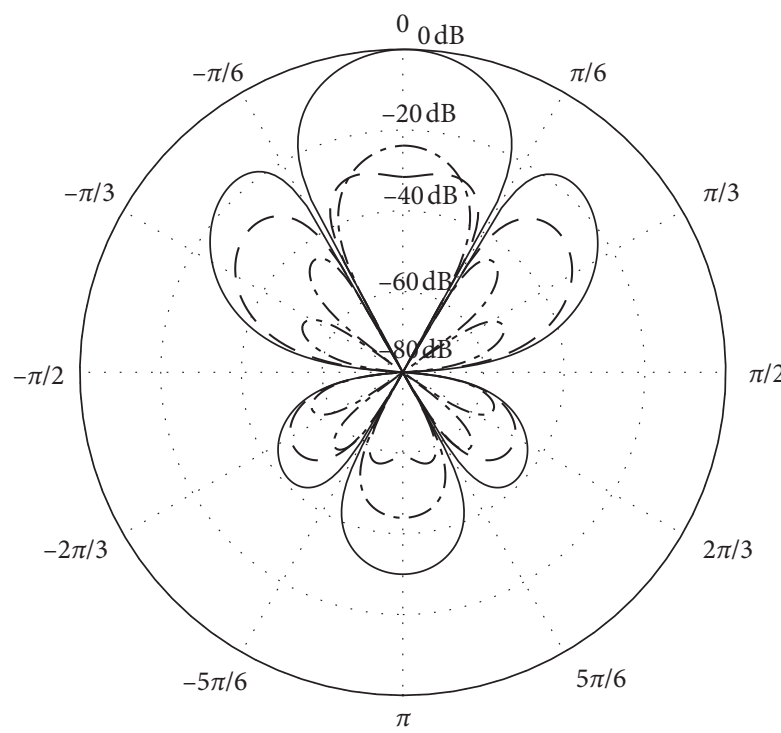

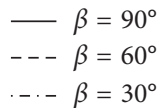

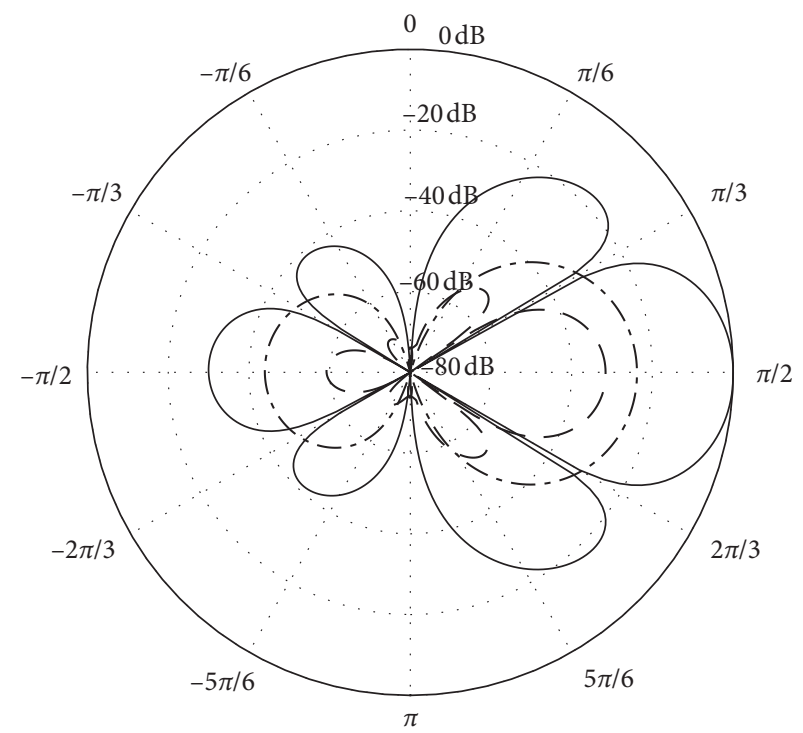

$-\alpha=0^{\circ}$

$--\alpha=30^{\circ}$

-. $\alpha=60^{\circ}$

(a)

(b)

FIgURE 4: URA beam pattern. (a) Horizontal pattern: $-\pi \leq \alpha \leq \pi$; (b) vertical pattern: $-\pi \leq \beta \leq \pi$.

ranges of the deviation angle. The slight difference of the power values corresponds to $16 / 15$, the ratio of antenna numbers. Figure 5(b) is the evaluation result for the $3 \mathrm{~dB}$ beamwidth for different values of $\theta$, showing the beamwidth remains relatively constant for all deviation angles.

Despite some detailed differences in their beam patterns, we observe from the comparison results that general beam characteristics for the two arrays are similar. Key features such as the beam peak power and the beamwidth do not depend on the type of the array, but on the number of elements in the array. Thus, the UTA with an appropriate number of elements can be an attractive alternate candidate of the URA to construct 3D antenna structures with expected beamforming characteristics. 


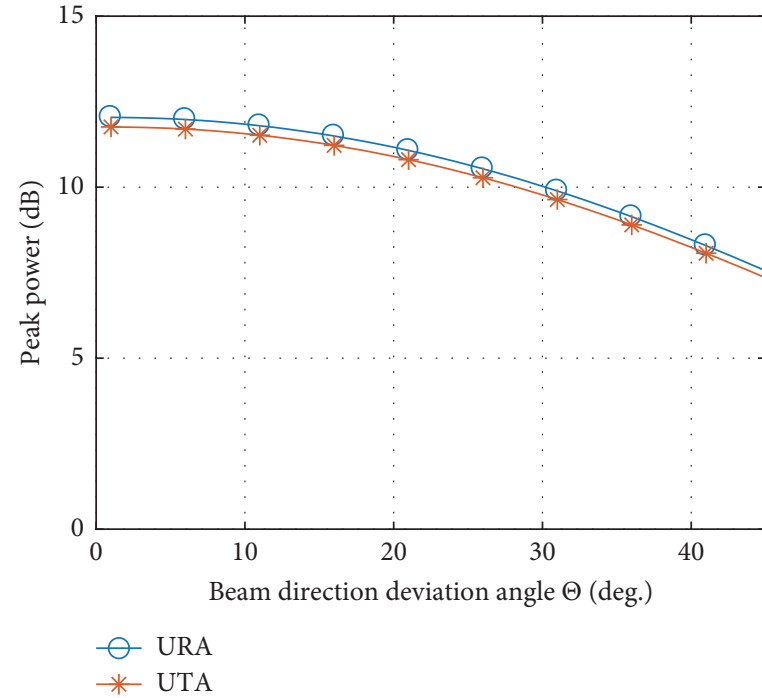

(a)

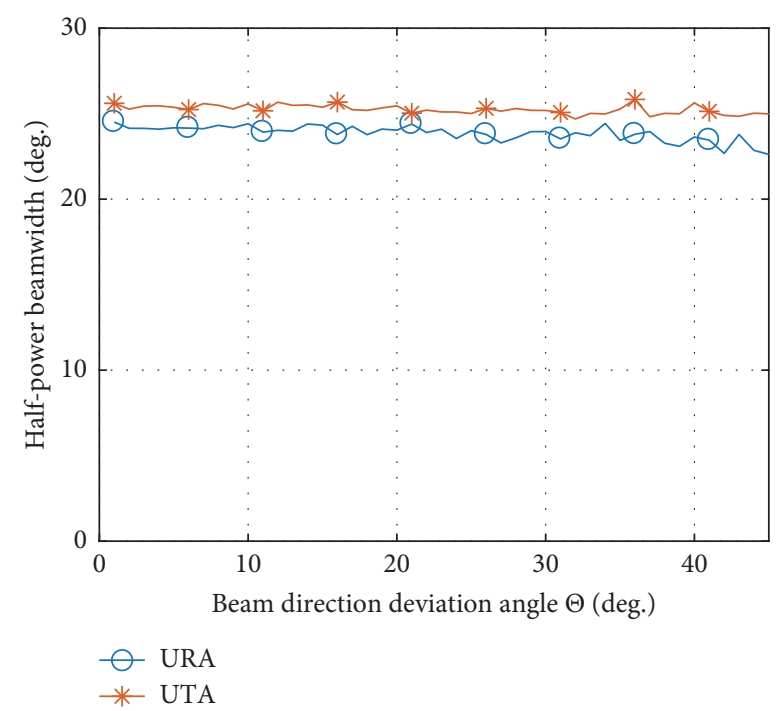

(b)

Figure 5: Beam pattern comparison. (a) Peak power and (b) half-power beamwidth.

We determine the set of beamforming vectors using the geometry of the antenna locations, which can be used for tilting transmitted beams from the UTA of an arbitrary size. Similar to the codevector generation for conventional twodimensional arrays, the determination of the beamforming vectors can be obtained by the Kronecker product of horizontal and vertical beams. Figure 6 shows the location indices for the antenna elements of the UTA. The antenna elements of the UTA exist in the subset of all possible locations within the rectangle produced by $L$ antenna rows and $2 L-1$ antenna columns. Let us first define the basic beamforming vectors for vertical beam generation. A vertical beamforming vector of length $L$ takes the form of the discrete Fourier transform (DFT) vector and is represented as

$$
\begin{aligned}
& \mathbf{a}_{p}=\left[\begin{array}{lllll}
1 & e^{j 2 \pi p / P} & e^{j 2 \pi 2 p / P} & \cdots & e^{j 2 \pi(L-1) p / P}
\end{array}\right]^{T} \\
& \text { for } p=0,1, \ldots, P-1 \text {, }
\end{aligned}
$$

with linearly increasing amount of phase values. Beam index $p$ determines one of the total $P$ angles for vertical beam tilting, and the $L \times P$ matrix

$$
\mathbf{A}=\left[\begin{array}{llll}
\mathbf{a}_{0} & \mathbf{a}_{1} & \cdots & \mathbf{a}_{P-1}
\end{array}\right],
$$

represents the set of $P$ vertical beamforming vectors. Second, the basic beamforming vectors for horizontal beam generation can be denoted by the vector of length $2 L-1$ as

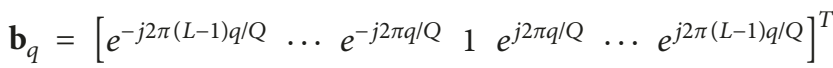

$$
\begin{aligned}
& \text { for } q=0,1, \ldots, Q-1 \text {, }
\end{aligned}
$$

which has a modified form of the DFT vector with the zero phase element in the centre of the vector. A horizontal beam is generated in one $Q$ tilting angle according to index $q$. By including all $Q$ beamforming vectors as columns of the $(2 L-1) \times Q$ matrix, we obtain

$$
\mathbf{B}=\left[\begin{array}{llll}
\mathbf{b}_{0} & \mathbf{b}_{1} & \cdots & \mathbf{b}_{\mathrm{Q}-1}
\end{array}\right] .
$$

To combine the vertical and horizontal beams, we multiply column vector $\mathbf{a}_{p}$ and row vector $\mathbf{b}_{q}^{T}$ to produce $L \times(2 L-1)$ codevector matrix in equation (11)

$$
\begin{aligned}
& \mathbf{C}_{p, q}=\mathbf{a}_{p} \mathbf{b}_{q}^{T}
\end{aligned}
$$

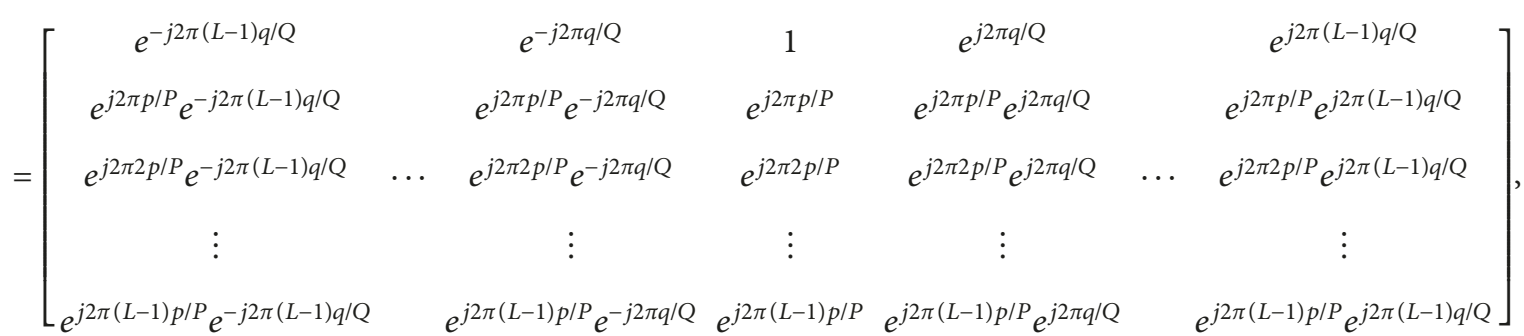




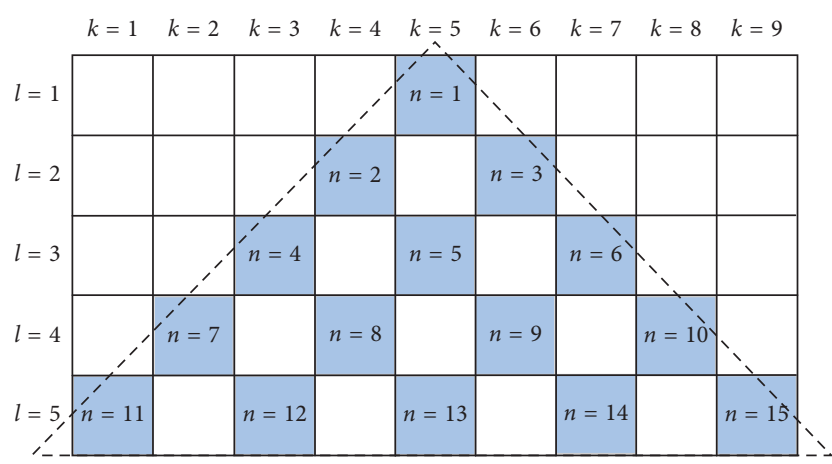

Figure 6: Antenna element indices for the UTA with $L$ rows and $2 L-1$ columns $(L=5)$.

for $p=0,1, \ldots, P-1$ and $q=0,1, \ldots, Q-1$. Note that $\mathbf{C}_{p, q}$ includes phase shifting terms for all elements in the rectangular area as shown in Figure 6. Beamforming vectors for the UTA are obtained by selecting the elements in equation (11) which correspond to the shaded elements of the triangular region in Figure 6, which are the UTA antenna elements. To choose $N$ elements out of all $L(2 L-1)$ elements in $\mathbf{C}_{p, q}$, we denote the $n$-th element of the beamforming vector for the UTA by $c_{p, q}^{n}$. This element is obtained by choosing $\mathbf{C}_{p, q}^{(l, k)}$, which is the $l$-th row and $k$-th column element in $\mathbf{C}_{p, q}^{p, q}$; i.e., we have the relation $\mathrm{c}_{p, q}^{n}=\mathbf{C}_{p, q}^{(l, k)}$. We need to specify how the codevector element index $n$ is related to matrix indices $l$ and $k$, and the relation is given by the following formulas

$$
\begin{aligned}
& l=x \quad \text { if } \frac{x(x-1)}{2}<n \leq x \frac{(x+1)}{2}, \\
& k=L-l^{2}+2 n-1 .
\end{aligned}
$$

Using these formulas, the codevector element index $n=1,2, \ldots, N-1$ is translated to the row index $l$ and column index $k$ and then the corresponding elements in equation (11) can be chosen. The codevector for the UTA is obtained by collecting selected elements $c_{p, q}^{n}$ as

$$
\mathbf{c}_{p, q}=\left[\begin{array}{llll}
c_{p, q}^{1} & c_{p, q}^{2} & \cdots & c_{p, q}^{N}
\end{array}\right]^{T} .
$$

Since there are $P$ vertical beam directions and $Q$ horizontal beam directions, the total of $P Q$ codevectors of length $N$ can be constructed by combining the beams in both directions. Thus, we include all these codevectors to form the codebook for the UTA as the $N \times(P Q)$ matrix

$$
\mathbf{C}=\left[\begin{array}{lllllllllllll}
\mathbf{c}_{1,1} & \mathbf{c}_{1,2} & \cdots & \mathbf{c}_{1, Q} & \mathbf{c}_{2,1} & \mathbf{c}_{2,2} & \cdots & \mathbf{c}_{2, Q} & \cdots & \mathbf{c}_{P, 1} & \mathbf{c}_{P, 2} & \cdots & \mathbf{c}_{P, Q}
\end{array}\right] .
$$

Beamforming vectors can be determined by choosing the column in equation (14) with the desired vertical beam index $p$ and horizontal beam index $q$.

To apply the beamforming vectors described above for coherent data transmission using multipanel arrays, an appropriate adjustment is required. Each UTA panel differs in its position and antenna boresight, and the correction parameter $\varphi_{k, m}$ needs to be multiplied to the chosen codevector to compensate for this difference as

$$
\mathbf{w}_{k, m}=\varphi_{k, m} \mathbf{c}_{p, q}
$$

to form the beamforming vector for the $m$-th panel and the $k$-th UE. To obtain the correction parameter, the distance from the reference point of each panel to the UE needs to be calculated. Without loss of generality, we let the location of the first element be the reference point for each UTA panel. The relative distance from $A_{m, 1}$ to the UE can be determined by taking the inner product of unit vector $\mathbf{u}=\overrightarrow{\mathrm{OU}} /|\overrightarrow{\mathrm{OU}}|$ from the origin to the UE and vector $\overrightarrow{\mathrm{OA}_{m}}$ in the direction of the reference point from the origin, i.e., $d_{m}=\mathbf{u} \cdot \overrightarrow{\mathrm{OA}_{m, 1}}$. The result of calculation using polar coordinates is given by [30]

$$
\begin{aligned}
d_{m}= & \mathbf{u} \cdot \overrightarrow{\mathrm{OA}_{m, 1}}=-r_{m, 1}\left(\sin \Theta \sin \theta_{m, 1} \cos \left(\Phi-\phi_{m, 1}\right)\right. \\
& \left.+\cos \Theta \cos \theta_{m, 1}\right)
\end{aligned}
$$

where $r_{m, 1}$ is the distance between the origin and the reference antenna location. Using this information, the channel phase difference for the reference antenna element in each panel can be obtained as

$$
\begin{aligned}
\angle \varphi_{k, m} & =\frac{2 \pi f_{c} d_{m}}{c} \\
& =-2 \pi r_{m, 1} \frac{\left(\sin \Theta \sin \theta_{m, 1} \cos \left(\Phi-\phi_{m, 1}\right)+\cos \Theta \cos \theta_{m, 1}\right)}{\lambda},
\end{aligned}
$$

where $f_{c}$ is the carrier frequency, $c$ is the propagation speed, and $\lambda$ is the wavelength. The correction parameter in equation (15) is determined by exponentiating the negative value of equation (17) to compensate for the phase difference as

$$
\varphi_{k, m}=e^{-j \angle \varphi_{k, m}}
$$

We can obtain the correction parameter for each panel by using the coordinate information of the reference antenna and the target UE from equation (18) and apply it to transmit beams from the given multipanel array structure.

\section{Multipanel UTAs}

Multiple UTAs can be combined together to construct 3D arrays of various shapes. All UTAs are of the same equilateral triangular shape, and they can be used as the basic building blocks for more complex and versatile antenna structures. For example, the UTAs can be put together to form a multifaceted pyramid which can be either wall-mounted or attached to the ceiling. As more small cells are being deployed for $5 \mathrm{G}$ NR, such ceiling type of antenna arrays fit well for indoor small cell applications. Figure 7 is an illustrative example of using multiple UTAs to form the pyramid shaped 3D array, mounted to the indoor ceiling for efficient signal transmission to the receivers within the cell. Parameters $\Theta_{1}$, $\Theta_{2}$, etc. are the beam deviation angles from their respective panel boresight. The signal to the target UE can 


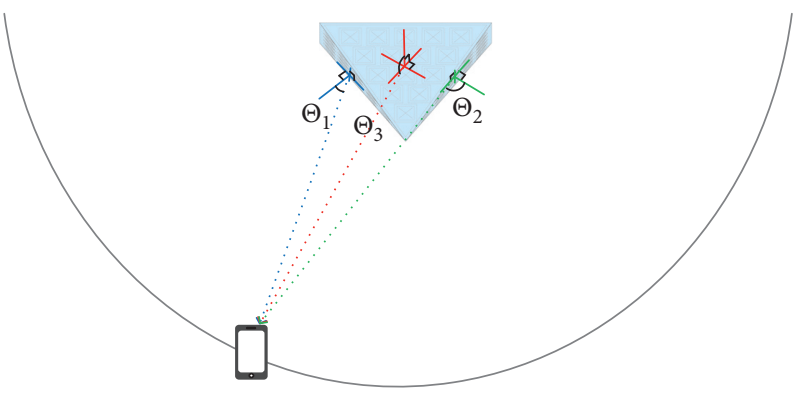

Figure 7: Antenna array for indoor small cell transmission.

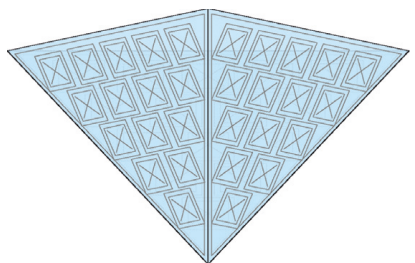

(a)

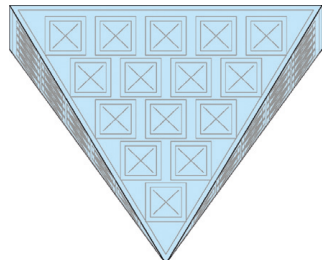

(b)

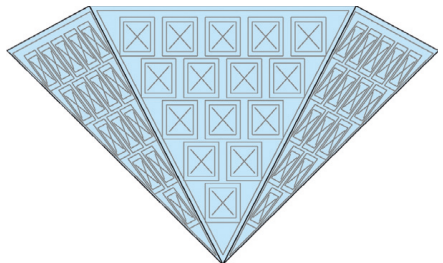

(c)

Figure 8: Ceiling-type 3D antenna arrays. (a) $M=3$; (b) $M=4$; (c) $M=5$.

be transmitted from the panel with the smallest deviation angle only or from multiple panels using the beam vectors for coherent signal combining. Adding more UTA panels results in 3D array structures in different shapes. Figure 8 shows ceiling-type arrays for the number of panels $M=3$, 4 , and 5. For these arrays, the angle between the boresight of the UTA panels and the direction perpendicular to the ceiling surface is $70.5^{\circ}, 54.7^{\circ}$, and $37.4^{\circ}$, respectively. When the number of panels increases to $M=6$, the array lies flat to the ceiling surface and the panel boresight is the direct downward direction. Depending on the room geometry and the typical UE distribution, a particular type of 3D array can be chosen for enhanced transmission performance.

The ceiling-type arrays introduced in Figure 8 have the limitation of covering only one hemisphere of the entire 3D space. To overcome this limitation, we can construct array structures based on regular polyhedrons and their variations to include more UTA panels covering the whole space. Figure 9 shows such array structures using $M=8,20,60$, and 80 panels. The first two arrays in Figures 9(a) and 9(b) are constructed by attaching a UTA panel to each face of the octahedron and icosahedron, respectively. Dodecahedron with 12 faces cannot be directly used to construct an array with UTAs since its faces are pentagons. Instead, we apply 5 UTAs to cover each face of the dodecahedron as shown in Figure 9(c) and obtain the 3D structure with 60 UTA panels. Also, each triangular face of the icosahedron can be further divided to include four equilateral triangles in a smaller size, providing the installation space for 80 UTA panels as illustrated in Figure 9(d). The total number of antenna elements is determined as $M_{T}=M N$, where $N$ is the number of antennas in each panel. Using $N=15$ elements for the UTA, the number of total antennas in the array structure with
$M=60$ panels amounts to $M_{T}=900$. Hence, massive arrays in various shapes and sizes can be built by multiple UTAs by changing the design parameters. As the demand for $3 \mathrm{D}$ antenna arrays increases, the mass production of UTAs provided at a reduced cost can be used for the array construction.

\section{Performance Evaluation}

We evaluate the performance of the 3D arrays for several different transmission scenarios. Here, we are interested in judging the applicability of the candidate arrays to the UEs in the $3 \mathrm{D}$ space when the direct line-of-sight (LoS) beam is the dominating component of transmission. Thus, only the normalized received power of the LoS beam is measured based on the deviation angle from each panel to the UE, ignoring the effects of the path loss or the multipath signals. The normalized received power has the unit value when an antenna element is directly pointed to the target UE with the zero deviation angle. For the UTA with $M$ elements, the received signal is combined to exhibit the normalized received power of $10 \log _{10} M \mathrm{~dB}$ for the UE with the zero deviation angle. The received power decreases as the deviation angle becomes larger, and the exact amount of the decrease is determined by the gain pattern described in equation (6). When $M$ panels are collaboratively transmitting the beam to the UE in a given location, we measure the deviation angle of all panels and arrange them in ascending order as $\Theta_{1}<\Theta_{2}<\cdots<\Theta_{M}$. The corresponding normalized received power can be then listed in descending order $P_{1}>P_{2}>\cdots>P_{M}$. The subscript index $k$ in variables $\Theta_{k}$ and $P_{k}$ indicates the order of contribution for the respective panel. For example, $k=2$ suggests that $\Theta_{2}$ is the second smallest deviation angle among all panels, 


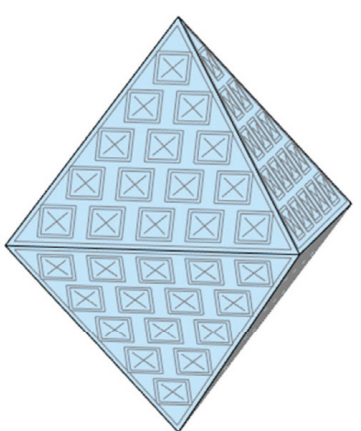

(a)

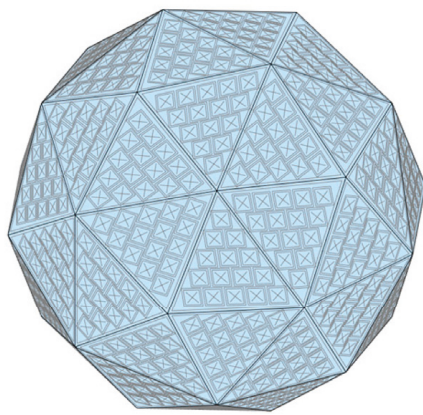

(c)

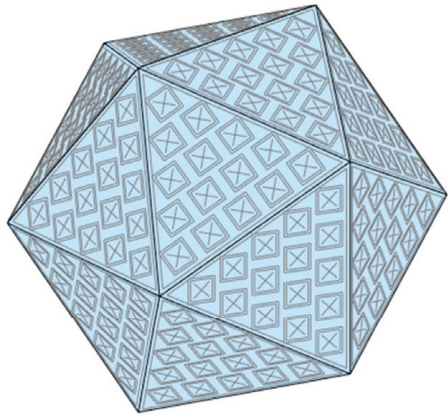

(b)

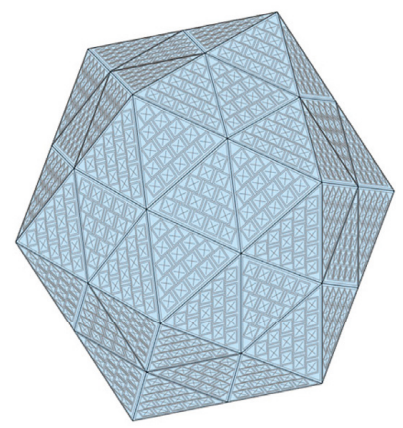

(d)

Figure 9: Polyhedron-type 3D antenna arrays. (a) $M=8$; (b) $M=20$; (c) $M=60$; (d) $M=80$.

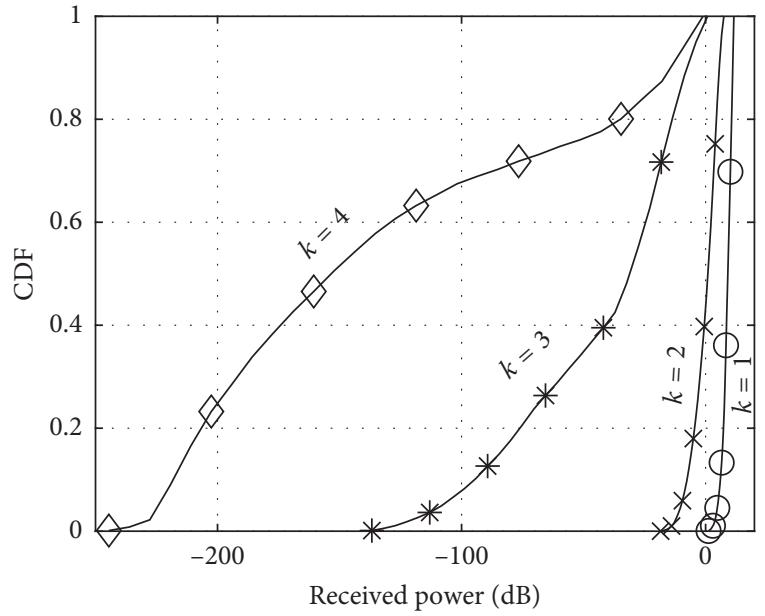

(a)

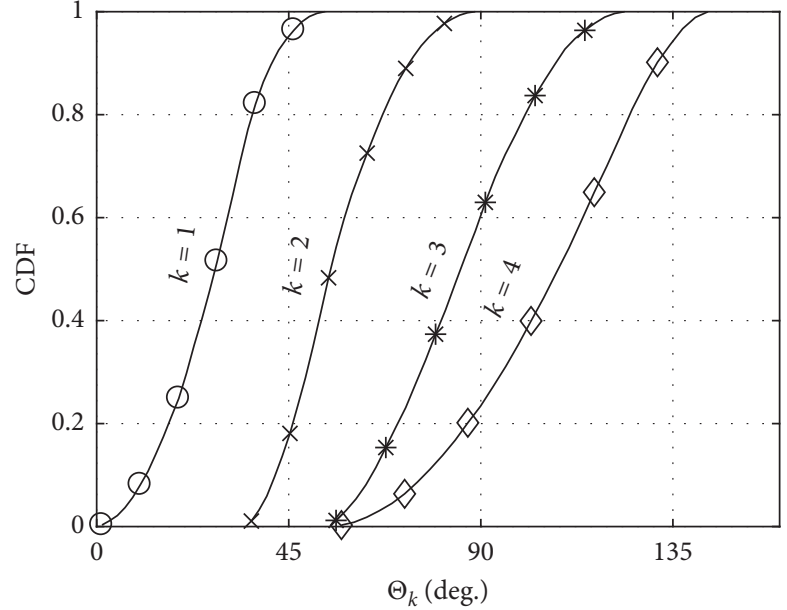

(b)

FIGURE 10: Transmission characteristics for the ceiling-type 3D array using $M=4$ UTAs. (a) Received power and (b) deviation angle.

providing the second largest normalized received power $P_{2}$ to the UE.

Figure 10 shows the transmission characteristics of the ceiling-type 3D array described in Figure 7 with $M=4$ panels, to the UE which is assumed to be located uniformly over the hemisphere. In Figure 10(a), the cumulative distribution function (CDF) of the normalized received power is shown for beams from four panels. The distribution of the largest received power $P_{1}$ is indicated by $k=1$ in the figure, with the average value of $8.9 \mathrm{~dB}$. The average values of $P_{2}, P_{3}$, and $P_{4}$ are respectively $0.3,-15.1$, and $-16.6 \mathrm{~dB}$, suggesting the amount of received power rapidly decreases for panels with larger deviation angles. For $k>2$, the amount of power contribution is significantly smaller than the first two panels. Therefore, using up to two panels for collaborative transmission seems to suffice in this scenario. Figure 10(b) shows the CDF of $\Theta_{k}$, indicating larger deviation angle distributions as $k$ increases. The average values of $\Theta_{k}$ are 28.1, 57.5, 87.0 , and $107.8^{\circ}$ for $k=1,2,3$, and 4 . The average values for $\Theta_{3}$ and $\Theta_{4}$ far exceed the $3 \mathrm{~dB}$ beamwidth of the gain pattern in equation (6), providing a negligible power contribution to the target UE. 


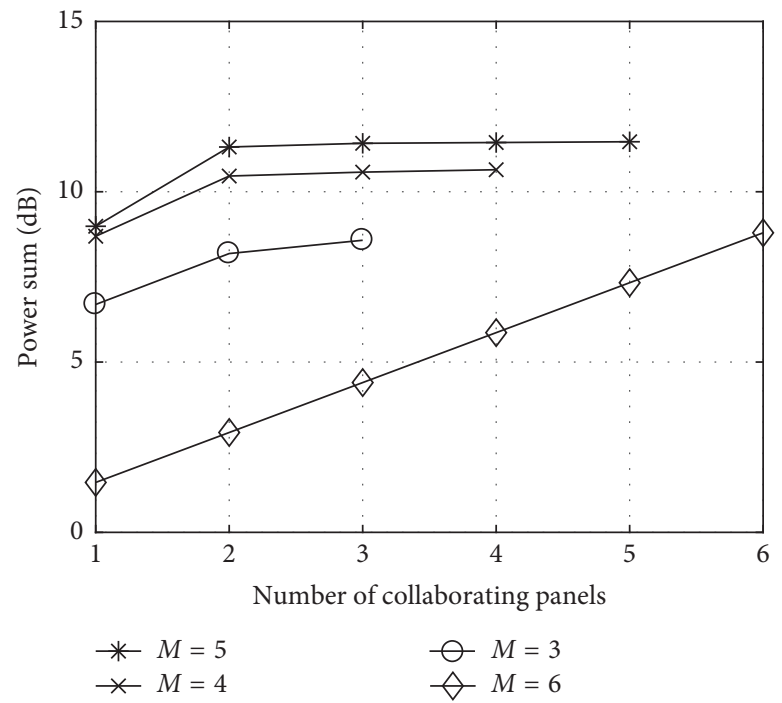

FIGURE 11: Received power versus the number of collaborating panels for the ceiling-type 3D arrays.

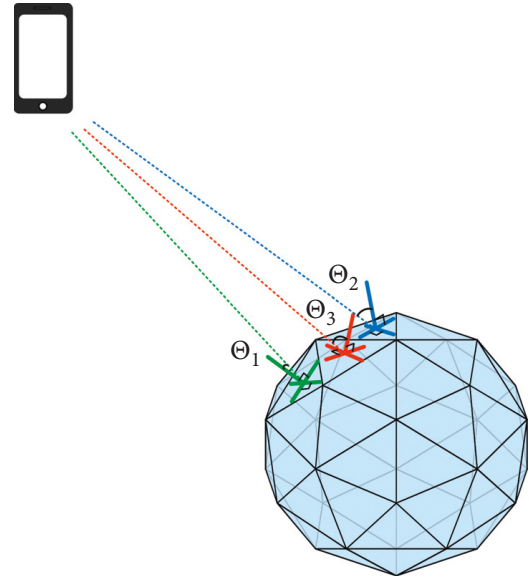

(a)

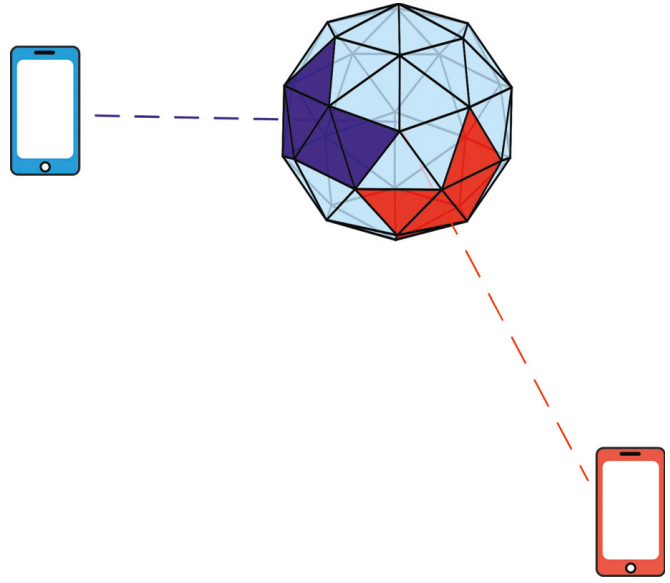

(b)

FIgURE 12: Transmission using the 3D array. (a) Single-user transmission and (b) multiuser transmission.

We apply the ceiling-type arrays with $M=3,4,5$, and 6 UTA panels to determine the sum of normalized received power for the UE in a random location over the hemisphere. In Figure 11, the power sum is shown for the increasing number of collaborating panels $M^{\prime}$ performing coherent signal combining. It can be observed in the figure that the power becomes saturated for $M^{\prime}=2$, and using more panels does not provide additional power gain. Only exception to this is the array with $M=6$ UTA panels, for which all panel surfaces lie flat to the same surface. In this case, the deviation angle for all panels to the UE is identical and the received power increases linearly as $M^{\prime}$ increases. It is also observed from the figure that the array with $M=5$ UTA panels provides the largest normalized power, suggesting its structural advantage of efficiently covering the space in terms of the panel boresight to random UEs.

Next, the normalized received power using the polyhedron-based $3 \mathrm{D}$ array with $M=60$ panels shown in
Figure $9(\mathrm{c})$ is considered. For this case, we assume beamforming is performed to random UEs located over the spherical model with the array in the centre, covering the whole space. We first evaluate the normalized received power for the single-user transmission scenario depicted in Figure 12(a), and then extend the result to examine the sum rate for the multiuser transmission scenario indicated in Figure 12(b).

Figure 13 shows the CDFs for the normalized received power and the deviation angle. Although as many as 60 panels can be collaboratively used for the single-user transmission, up to approximately 5 panels contribute to the most of the received power. In Figure 13(a), the power distribution is plotted for $k=1$ to 5 , for which the average values are 11.3, 10.9, 10.0, 9.4, and $8.7 \mathrm{~dB}$ in descending order. Unlike the ceiling-type arrays, the power decrease is less severe for increasing values of $k$. This can also be observed in the distribution of the deviation angles shown in 


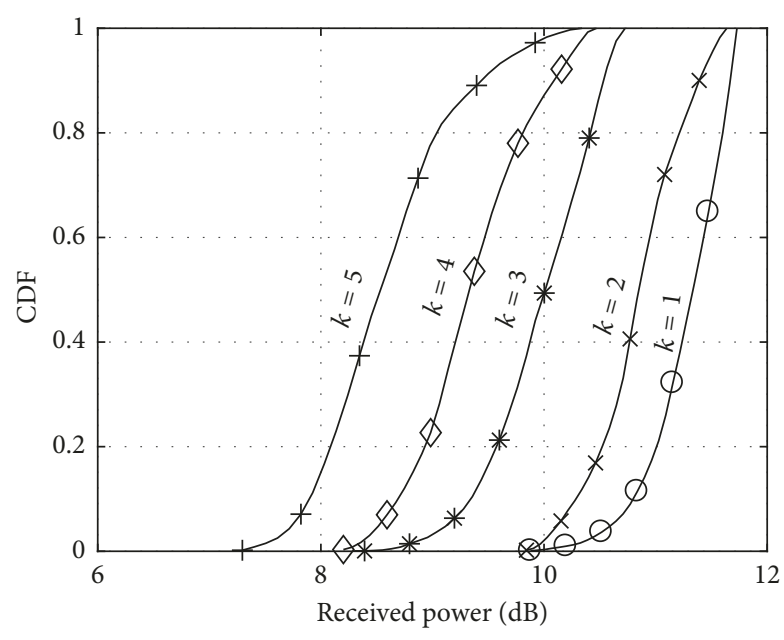

(a)

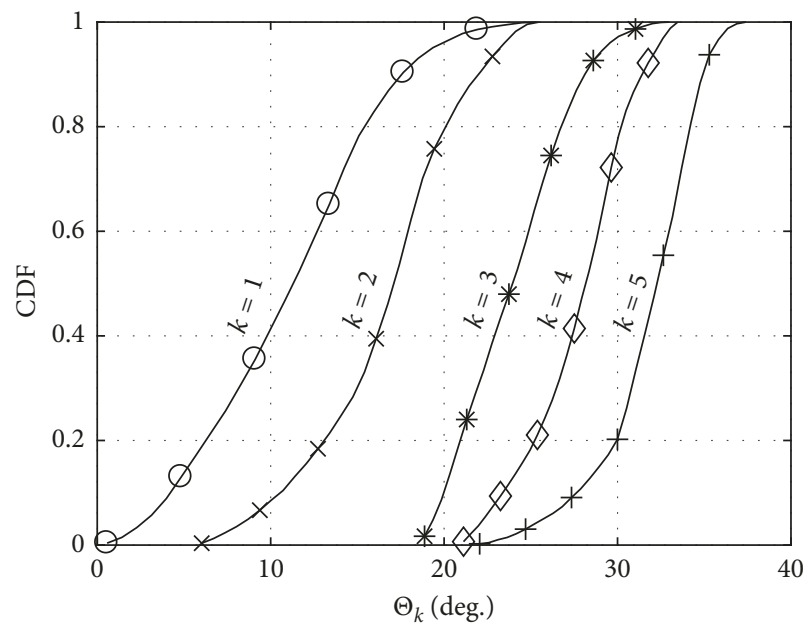

(b)

FIgURE 13: Transmission characteristics for the polyhedron-type 3D array using $M=60$ UTAs. (a) Received power; (b) deviation angle.

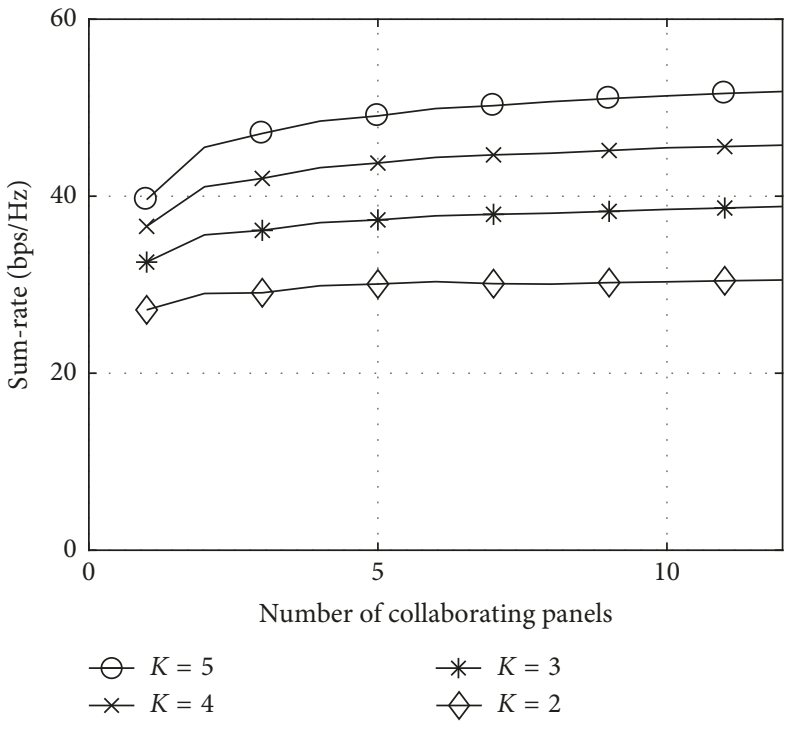

(a)

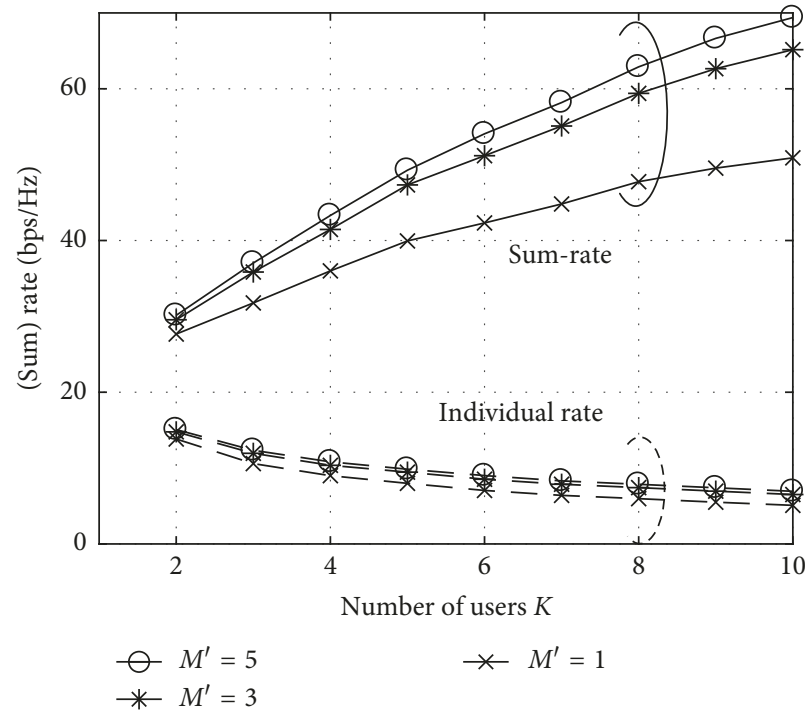

(b)

Figure 14: Performance of multiuser transmission for the polyhedron-type 3D array using $M=60$ UTAs. (a) Sum rate vs. number of collaborating panels; (b) rate vs. number of users.

Figure 13(b), with the average vales of $11.5,16.9,24.2,28.0$, and $32.0^{\circ}$. For the spherical-shaped array with a large number of panels, more panels with reasonably small deviation from the boresight contribute in transmitting signals to the target UE.

The array provides the beam transmission capability to all isotropic UE locations in the 3D space, and only the limited number of panels is sufficient for beamforming to a single user. Therefore, multiuser transmission to geometrically separated UEs is desired for enhanced utilization of the array resource to increase the system throughput. We randomly locate $K$ UEs over the space and assign a set of panels for beamforming to a given UE. Figure 10(b) is an illustration of multiuser transmission to $K=2$ UEs, where the panels assigned for the UE in the left are indicated in blue colour and the panels for the UE in the downward direction are marked in red. The panel allocation strategy is to assign panels to UEs in a round-robin fashion, with the panel having the boresight closest to the direction of the UE assigned in turns. If the panel with such a condition is already assigned to some other UE, the panel with the next smallest deviation angle is assigned. The procedure continues until all UEs are assigned with $M^{\prime}$ panels for their data transmission, where $M^{\prime}$ is the predetermined number of panels used for collaborative beamforming to each user.

We apply this panel allocation process to $K$ multiple UEs using the polyhedron-based array with 60 UTA panels to evaluate the sum-rate performance. The sum rate is determined by adding the individual rate of the UEs as 
described in equation (5). The individual rate is obtained by the capacity formula, where the signal-to-interference ratio is computed by the received power from the panels assigned to the UE and the interference power from the panels for the other UEs. In Figure 14(a), the sum rate is evaluated from repeated simulations of randomly located UEs when the number of simultaneously present UEs for beam transmission is $K=2,3,4$, and 5. An increasing value of the sum rate is shown as $K$ becomes large, showing the multiuser transmission capability of the array. It is shown in the figure that the saturation does not occur until $M^{\prime}=10$ collaborating panels, suggesting 10 or more panels can be assigned for each UE. However, the additional gain after using $M^{\prime}=5$ panels becomes limited, and using more panels will incur increased operation complexity and power consumption. In Figure 14(b), we evaluate the rates when the number of UEs increases, while using 5 or less panels for each UE. Although the individual rate tends to exhibit a slow decrease for more UEs, the sum rate keeps increasing up to 10 UEs, confirming the applicability of the array to multiuser beamforming.

\section{Conclusions}

Antenna arrays in diverse forms and structures are desired for enhanced beamforming functionalities, and the utilization of triangular panels and their combinations to construct 3D arrays has been suggested. The UTAs provide the geometrical advantage over the rectangular counterpart to build various 3D array structures, with possible collaboration of multiple panels for the increased signal strength to the target user. The beam-tilting capability via phase shifting from each panel can also be provided by the proposed codebook for the limited feedback channels. Transmission using the $3 \mathrm{D}$ arrays is especially effective for the multiuser scenario, where a large number of users can be simultaneously supported with the improved system throughput.

\section{Data Availability}

The simulation data used to support the findings of this study are available from the corresponding author upon request.

\section{Conflicts of Interest}

The authors declare that they have no conflicts of interest.

\section{Acknowledgments}

This work was supported by the National Research Foundation (NRF) of Korea, MSIP (NRF-2017R1A2B4002367), and by LG Electronics.

\section{References}

[1] X. Ge, S. Tu, G. Mao, C. Wang, and T. Han, "5G ultra-dense cellular networks," IEEE Wireless Communications, vol. 23, no. 1, pp. 72-79, 2016.

[2] V. Raghavan, A. Partyka, A. Sampath et al., "Millimeter-wave MIMO prototype: measurements and experimental results,"
IEEE Communications Magazine, vol. 56, no. 1, pp. 202-209, 2018.

[3] H. Tullberg, P. Popovski, Z. Li et al., "The METIS 5G system concept: meeting the $5 \mathrm{G}$ requirements," IEEE Communications Magazine, vol. 54, no. 12, pp. 132-139, 2016.

[4] M. Xiao, S. Mumtaz, Y. Huang et al., "Millimeter wave communications for future mobile networks," IEEE Journal on Selected Areas in Communications, vol. 35, no. 9, pp. 1909-1935, 2017.

[5] J. Zhang, X. Ge, Q. Li, M. Guizani, and Y. Zhang, "5G millimeter-wave antenna array: design and challenges," IEEE Wireless Communications, vol. 24, no. 2, pp. 106-112, 2017.

[6] J. G. Andrews, T. Bai, M. N. Kulkarni, A. Alkhateeb, A. K. Gupta, and R. W. Heath Jr., "Modeling and analyzing millimeter wave cellular systems," IEEE Transactions on Communications, vol. 65, no. 1, pp. 403-430, 2017.

[7] L. Zhou and Y. Ohashi, "Fast codebook-based beamforming training for mmWave MIMO systems with subarray structures," in Proceedings of the Vehicular Technology Conference (VTC2015-Fall), pp. 1-5, Boston, MA, USA, September 2015.

[8] E. Onggoanusi, M. S. Rahman, L. Guo et al., "Modular and high-resolution channel state information and beam management for $5 \mathrm{G}$ new radio," IEEE Communications Magazine, vol. 56, no. 3, pp. 48-55, 2018.

[9] 3GPP TS 36.211 V10.0.0, "Evolved universal terrestrial radio access (E-UTRA)," Physical Channels and Modulation, 2010.

[10] 3GPP TS 36.213 V14.1.0, "Evolved universal terrestrial radio access (E-UTRA)," Physical Layer Procedures, 2016.

[11] T. Thomas and F. Vook, "Improving the performance of the UCA when using LTE codebook feedback," in Proceedings of IEEE Wireless Communications and Networking Conference (WCNC), pp. 847-851, Shanghai, China, April 2012.

[12] L. Wu, H. Yang, and D. Wang, "Hadamard transform based codebook design for uniform circular arrays in mobile radio communications," in Proceedings of IEEE Vehicular Technology Conference (VTC Fall), pp. 1-5, Quebec City, Canada, September 2012.

[13] W. M. Dorsey, R. Mital, and D. P. Scholnik, "Phase-only synthesis of omnidirectional patterns with multiple nulls from a uniform circular array," in Proceedings of IEEE International Symposium on Antennas and Propagation (APSURSI), pp. 765-766, Fajardo, Puerto Rico, July 2016.

[14] Q. Nadeem, A. Kammoun, M. Debbah, and M. Alouini, "Spatial correlation characterization of a uniform circular array in 3D MIMO systems," in Proceedings of the IEEE International Workshop on Signal Processing Advances in Wireless Communications SPAWC'16, pp. 1-6, Edinburgh, UK, July 2016.

[15] H. Jiacai, S. Yaowu, and T. Jianwu, "Joint estimation of DOA, frequency, and polarization based on cumulants and UCA," Journal of Systems Engineering and Electronics, vol. 18, no. 4, pp. 704-709, 2007.

[16] R. Chen, W. Yang, H. Xu, and J. Li, "A 2-D FFT-based transceiver architecture for OAM-OFDM systems with UCA antennas," IEEE Transactions on Vehicular Technology, vol. 67, no. 6, pp. 5481-5485, 2018.

[17] L. Zuo, J. Pan, and Z. Shen, "Analytical algorithm for 3-D localization of a single source with uniform circular array," IEEE Antennas and Wireless Propagation Letters, vol. 17, no. 2, pp. 323-326, 2018.

[18] S. Zhu, Y. Yang, Y. Wang, Z. He, and Q. Yang, "Beamforming with a line array mounted on an infinite rigid cylindrical baffle," in Proceedings of the IEEE International Conference on 
Signal Processing, Communications and Computing (ICSPCC), pp. 1-5, Xiamen, China, January 2017.

[19] S. M. Razavizadeh, M. Ahn, and I. Lee, "Three-dimensional beamforming: a new enabling technology for $5 \mathrm{G}$ wireless networks," IEEE Signal Processing Magazine, vol. 31, no. 6, pp. 94-101, 2014.

[20] H. Ahn, B. Tomasic, and S. Liu, "Digital beamforming in a large conformal phased array antenna for satellite operations support-architecture, design, and development," in Proceedings of the IEEE International Symposium on Phased Array Systems and Technology, pp. 423-431, Waltham, MA, USA, October 2010.

[21] M. Henderson, M. B. Davis, and M. Huisjen, "GDPAA advanced technology demonstration overview and results," in Proceedings of the International Symposium on Phased Array Systems and Technology, pp. 140-143, Waltham, MA, USA, October 2010.

[22] E. Guangxi and H. Yang, "Design and test of geodesic dome phased array system for multi-target TT\& C and communication," in Proceedings of the International Conference on Optical Communications and Networks (ICOCN), pp. 1-3, Hangzhou, China, September 2016.

[23] M. A. Salas-Natera, R. M. Rodriguez-Osorio, and L. de Haro, "Procedure for measurement, characterization, and calibration of active antenna arrays," IEEE Transactions on Instrumentation and Measurement, vol. 62, no. 2, pp. 377-391, 2013.

[24] D. Zhu, B. Li, and P. Liang, "A novel hybrid beamforming algorithm with unified analog beamforming by subspace construction based on partial CSI for massive MIMO-OFDM systems," IEEE Transactions on Communications, vol. 65, no. 2, pp. 594-607, 2017.

[25] F. Sohrabi and W. Yu, "Hybrid digital and analog beamforming design for large-scale antenna arrays," IEEE Journal of Selected Topics in Signal Processing, vol. 10, no. 3, pp. 501-513, 2016.

[26] J.-S. Sheu, "Hybrid digital and analogue beamforming design for millimeter wave relaying systems," Journal of Communications and Networks, vol. 19, no. 5, pp. 461-469, 2017.

[27] K. A. R. G. Chandra Mouli, M. S. Anuradha, D. Kuppili, and D. Gopi, "Analysis of rectangular and triangular microstrip antenna arrays using HFSS," in Proceedings of the International Conference on Electromagnetic Interference and Compatibility (INCEMIC), pp. 25-31, Visakhapatnam, India, July 2015.

[28] Y. S. H. Khraisat and M. M. Olaimat, "Comparison between rectangular and triangular patch antennas array," in Proceedings of the International Conference on Telecommunications (ICT), pp. 1-5, Jounieh, Lebanon, April 2012.

[29] 3GPP TR 36.873 V12.2.0, Study on 3D Channel Model for LTE, 2015.

[30] C. B. Dietrich, "Diversity antenna configurations for handheld wireless communication terminals," Ph.D. Dissertation, Virginia Polytechnic Institute and State University, Blacksburg, VA, USA, 2000. 


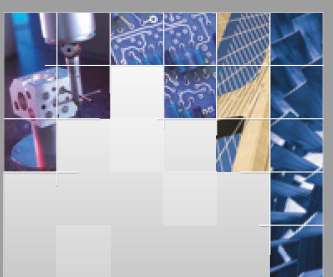

\section{Enfincering}
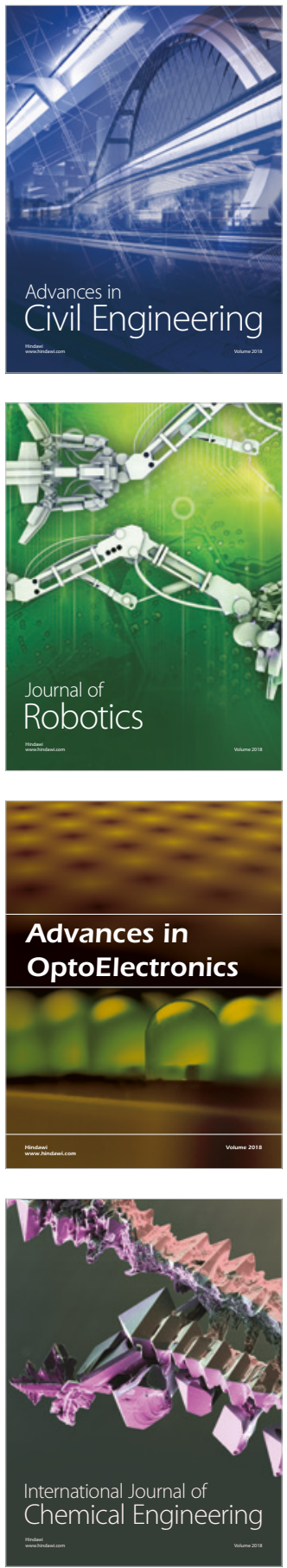

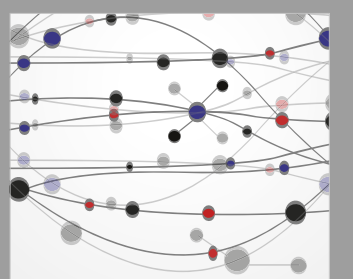

\section{Rotating \\ Machinery}

The Scientific World Journal

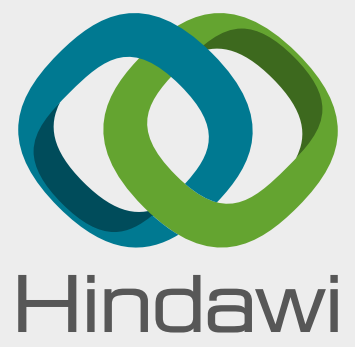

Submit your manuscripts at

www.hindawi.com
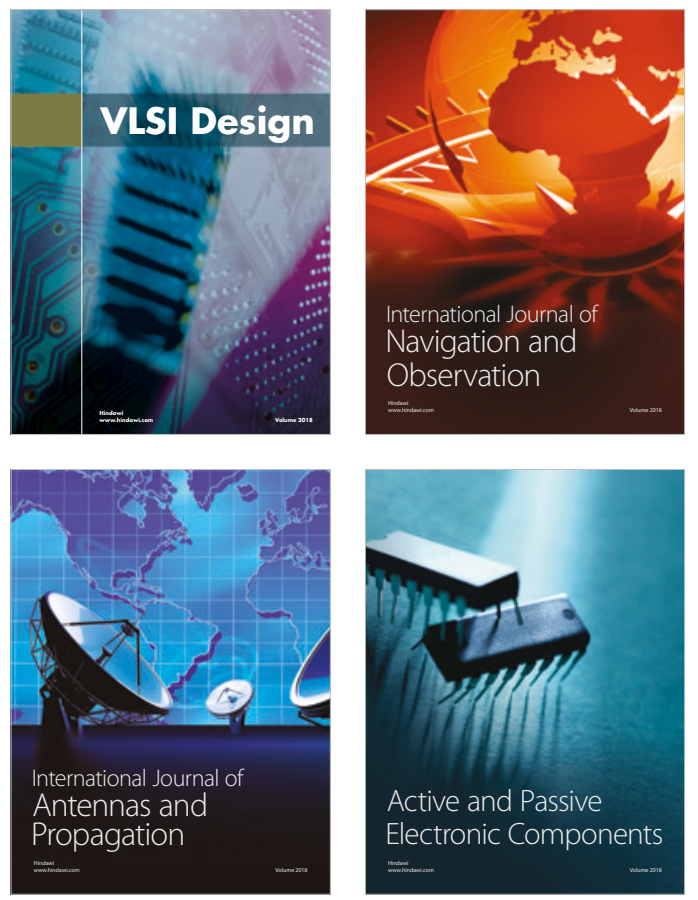
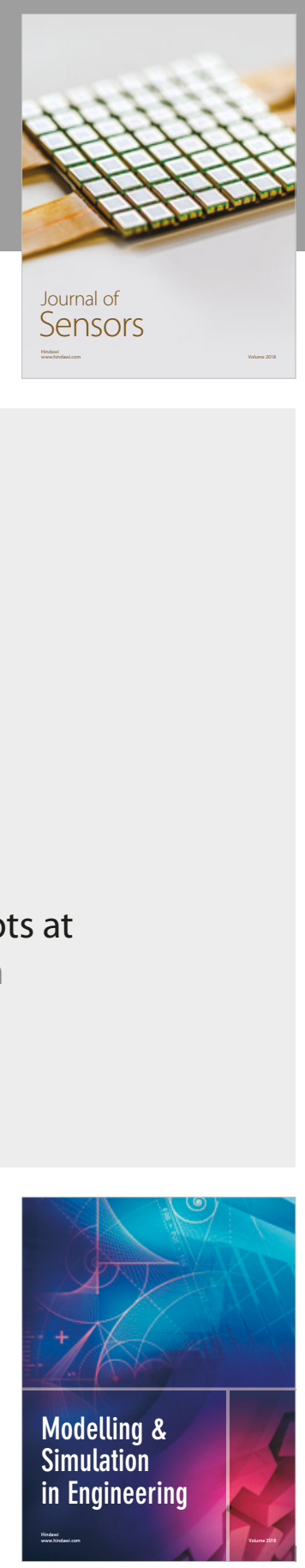

\section{Advances \\ Multimedia}
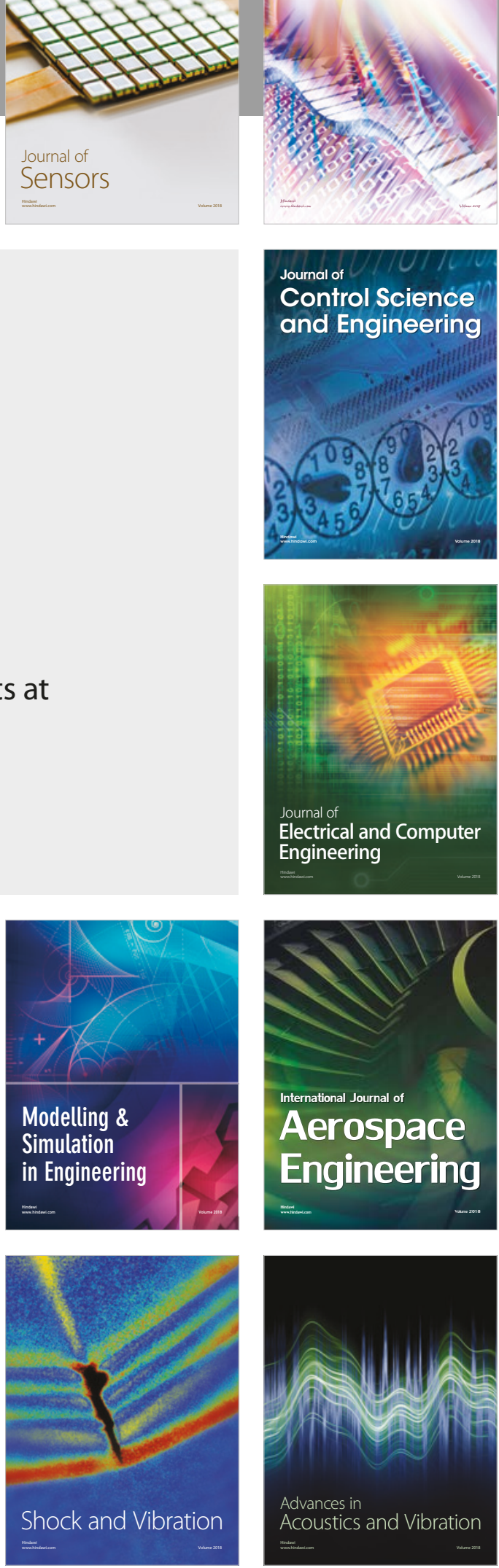